\title{
Conflitos familiares abordados na terapia comunitária integrativa
}

\author{
Family conflicts approached in integrative community therapy
}

Conflictos familiares abordados en la terapia comunitaria integrativa

\author{
Aralinda Nogueira Pinto de Sá1, Ianine Alves da Rocha², Marina Nascimento de Moraes ${ }^{3}$ \\ Lucineide Alves Vieira Braga ${ }^{4}$, Maria Oliveira Ferreira Filha ${ }^{5}$, Maria Djair Dias ${ }^{6}$
}

\section{RESUMO}

O presente estudo objetivou realizar um levantamento sobre conflitos familiares, identificando os principais motivos relacionados e as estratégias de enfrentamento. Trata-se de pesquisa documental, desenvolvida no município de João Pessoa, que utilizou o banco de dados de registros das rodas de Terapia Comunitária realizadas no período de julho de 2008 a dezembro 2009. Foram analisadas 775 fichas de organização das informações, das quais 110 apresentavam o tema. Os dados foram submetidos a tratamento estatístico simples e interpretados à luz da literatura pertinente. Constatou-se a presença de conflitos entre cônjuges (48\%), pais/filhos (37\%), irmãos (8\%) e avós/netos (7\%), motivados pela desvalorização do papel da mulher, sobrecarga de trabalho, alcoolismo, separação, ciúmes, traição, problemas intergeracionais, violência e drogadição. Como estratégias de enfrentamento surgiram: autocontrole, autoconhecimento, suporte da comunidade, participação em grupos, diálogo e espiritualidade. A Terapia foi considerada um espaço de desabafo e alívio dos sofrimentos, fortalecendo vínculos, promovendo o empoderamento das famílias.

Descritores: Terapia; Família; Enfermagem Psiquiátrica.

\section{ABSTRACT}

The present study aimed at developing a survey regarding family conflicts, identifying the main related reasons and coping strategies. It consists of a documental study, developed in the municipality of João Pessoa, which used the database of records from community therapy groups that met during the period between July 2008 and December 2009. Analysis included 775 data organization charts, of which 110 presented the theme. Data were submitted to simple statistical treatment and interpreted in light of the pertinent literature. The study revealed the presence of conflict between spouses (48\%), parents/children (37\%), siblings ( $8 \%$ ) and grandparents/grandchildren (7\%), motivated by the underappreciation of the woman's role, work overload, alcoholism, separation, jealousy, betrayal, intergenerational problems, violence and drug addiction. The coping strategies that emerged were: self-control, self-knowledge, community support, participation in groups, dialogue and spirituality. The therapy was considered to be a space for alleviation of pain and relief of their suffering, strengthening bonds and promoting the empowerment of the families.

Descriptors: Therapy; Family; Psychiatric Nursing.

\section{RESUMEN}

Se objetivó efectuar relevamiento sobre conflictos familiares, identificando los principales motivos relacionados y las estrategias de enfrentamiento. Investigación documental desarrollada en municipio de João Pessoa, utilizando banco de datos de registros de las rondas de Terapia Comunitaria realizadas entre julio 2008 y diciembre 2009. Fueron analizadas 775 fichas de organización de informaciones, 110 de las cuales se referían al tema. Datos sometidos a tratamiento estadístico simple, interpretados de acuerdo a la literatura pertinente. Se constató la presencia de conflictos entre cónyuges (48\%), padres/hijos (37\%), hermanos (8\%) y abuelos/nietos (7\%); motivados por la desvalorización del papel femenino, sobrecarga laboral, alcoholismo, separación, celos, traición, problemas intergeneracionales, violencia y drogadicción. Como estrategias de enfrentamiento surgieron: autocontrol, autoconocimiento, soporte comunitario, participación en grupos, diálogo y espiritualidad. La Terapia fue considerada un espacio de desahogo y alivio de sufrimientos, fortaleciendo vínculos, promoviendo el empoderamiento de las familias.

Descriptores: Terapia; Familia; Enfermería Psiquiátrica.

\footnotetext{
${ }^{1}$ Enfermeira, Mestre em Enfermagem. Docente da Faculdade de Ciências Médicas da Paraíba. João Pessoa, PB, Brasil. E-mail: arallinda@yahoo.com.br.

${ }^{2}$ Enfermeira, Mestre em Enfermagem. João Pessoa, PB, Brasil. E-mail: ian ine@yahoo.com.br.

${ }^{3}$ Enfermeira. João Pessoa, PB, Brasil. E-mail: ninamoraes @hotmail.com.

${ }^{4}$ Enfermeira, Mestre em Enfermagem. Docente da Faculdade de Ciências Médicas da Paraíba. João Pessoa, PB, Brasil. E-mail: lucineide.avb@gmail.com.

5 Enfermeira, Doutora em Enfermagem. Professora Adjunto IV da Universidade Federal da Paraíba (UFPB). João Pessoa, PB, Brasil. E-mail: marfilha@yahoo.com.br.

${ }^{6}$ Enfermeira, Doutora em Enfermagem. Professora Adjunto IV da UFPB. João Pessoa, PB, Brasil. E-mail: mariadjair@yahoo.com.br.
} 


\section{INTRODUÇÃO}

A ideia de família remete a uma relação que envolve afetividade, harmonia e proteção de seus membros. Por ser o primeiro sistema que o indivíduo interage, ela forma a unidade básica da organização na sociedade e é pré-requisito de um sistema social estável, no qual cada membro tem uma posição e um papel socialmente definido(1).

O cenário familiar tem se modificado pelas crises nas sociedades decorrentes do capitalismo e responsáveis pelo surgimento de novas estruturas familiares, nas quais os papéis assumidos historicamente por seus membros estão sendo sacudidos pelas mudanças sociais, econômicas, culturais e educacionais(2).

As novas estruturas familiares têm sido caracterizadas pela redução do número de filhos, o retardo do matrimônio, o atraso da maternidade e o aumento da porcentagem de divórcios, implicando na formação de famílias mononucleares, com o número reduzido de seus componentes, e contribuindo para uma pluralidade de organizações. Esses indicadores definem as famílias dos países industrializados e em desenvolvimento, como é o caso do Brasil( ${ }^{(3)}$.

Um dos aspectos determinantes para a mudança das relações familiares foi a ascensão da figura feminina impulsionada pelo processo histórico de urbanização e industrialização, pelo qual a mulher contemporânea estabeleceu prioridades mais direcionadas ao mercado de trabalho em detrimento às atividades domésticas e à maternidade.

Apesar da ascensão do papel social da mulher não houve, paralelamente, a inversão do pensamento machista e a inclusão do homem nas atividades domésticas. Isto favoreceu a sobrecarga de trabalho feminino, o aumento do stress, desvio no padrão tradicional de hierarquia familiar, e consequentemente o surgimento de situações potencialmente geradoras de conflitos familiares, culminando algumas vezes na violência doméstica e relações permeadas pelo medo(4). Sendo assim, entende-se que grande parte dos conflitos familiares advém como consequência dessa conjuntura; no entanto, estas situações são condicionantes negligenciados de saúde que, frequentemente, não são discutidos como geradores de adoecimento mental.

O modelo biomédico, ainda fortemente presente no cuidado à saúde, não observa o contexto social, espiritual e cultural que o indivíduo se encontra, e, portanto, direciona conduta apenas para o controle das manifestações clínicas. Observa-se que, no cotidiano dos serviços de saúde, a rede de cuidados dispõe de poucos espaços para a fala e escuta dos sofrimentos dos indivíduos, das famílias e das comunidades, além de não oferecer infraestrutura para que as equipes de saúde possam realizar um atendimento mais integral aos usuários.

Por um lado existem alguns profissionais que não possuem uma sensibilidade para observar esta relação contextual, e por outro ainda tem as pessoas que buscam na terapia medicamentosa o alívio de sintomas físicos advindos da somatização dos conflitos de ordem familiar e social.

Entendendo o adoecimento como evento multicausal, percebe-se a necessidade de estratégias inovadoras no enfrentamento do sofrimento cotidiano. Nesse sentido, a Terapia Comunitária Integrativa (TCl) vem se apresentando como uma tecnologia de cuidado ou um procedimento terapêutico grupal, cuja finalidade é a promoção da saúde e a prevenção do adoecimento desenvolvida no âmbito da saúde mental.

Embora proporcione uma leveza informal, a metodologia da $\mathrm{TCl}$ encontra-se fundamentada na teoria da comunicação, na antropologia cultural, na teoria sistêmica, na pedagogia de Paulo Freire e na resiliência. São fundamentos que se complementam e constroem a identidade da Terapia Comunitária Integrativa perpassando por toda sua técnica, a qual acontece em cinco passos: acolhida, escolha do tema, contextualização, problematização e ritual de agregação(5).

Alguns estudos ${ }^{(6-10)}$ reconhecem o valor terapêutico da TCl na rede de atenção à saúde mental e como recurso de transformação da prática encaminhada para o modelo comunitário. É importante ressaltar que, a maioria das pesquisas foi elaborada por enfermeiras, o que demonstra a veemência da $\mathrm{TCl}$ no trabalho da categoria. Um estudo pioneiro ${ }^{(11)}$ sobre as repercussões da Terapia Comunitária Integrativa no cotidiano de seus participantes revelou que o sucesso desses encontros se deu pela abertura de um espaço de acolhimento para o sofrimento e angústias e alegrias, no qual oportuniza um processo de transformação que conseguiu fortalecer vínculos de amizade, comunitário e social. Deste estudo partiu uma sugestão de inserir a TCl como atividade dentro de alguma política pública ${ }^{(11)}$. Em 2010 a TCI foi 
incorporada como um projeto especial na Política das Práticas Integrativas e Complementares, se firmando como mais um valioso instrumento de cuidado em saúde mental.

Outras pesquisas ${ }^{(8,12-13)}$ apontam os impactos positivos da $\mathrm{TCl}$ no enfrentamento das situações adversas da vida cotidiana, se mostrando fomentadora de empoderamento, resiliência, autoconfiança e fé(13). Isso porque os participantes partilham tanto seus problemas como as estratégias de enfrentamento dos sofrimentos, possibilitando aos indivíduos, famílias e grupos, desenvolverem a autonomia, fortalecerem os vínculos, e adquirirem as bases necessárias para o equilíbrio pessoal e social(5).

Mediante o exposto, surgiram os seguintes questionamentos: Quais conflitos familiares foram mais abordados nas Terapias Comunitárias Integrativas? Quais as estratégias de enfrentamento utilizadas pelas famílias para lidar com esses conflitos?

A relevância desse estudo consiste em divulgar uma tecnologia inovadora, que atua como uma tecnologia leve de cuidado na unidade familiar e de empoderamento na conduta dos profissionais de saúde, a Terapia Comunitária Integrativa. Dessa maneira, este estudo teve como objetivo analisar os conflitos familiares abordados nas Terapias Comunitárias Integrativas e descrever as estratégias de enfrentamento utilizadas pelas famílias para lidar com seus conflitos.

\section{METODOLOGIA}

Trata-se de um estudo retrospectivo, do tipo documental, o qual analisou fontes que não receberam nenhum tratamento científico. A pesquisa foi realizada no banco de dados sobre a Terapia Comunitária Integrativa, armazenado no Polo Formador Movimento Integrado de Saúde Comunitária da Paraíba (MISC-PB). A fonte principal foram as Fichas de organização das informações da $\mathrm{TCl}$, realizadas no período de julho de 2008 a dezembro de 2009, nas quais foram registrados rudimentos feitos pelos 60 terapeutas comunitários, acerca de cada roda realizada, a exemplo de: número de participantes, faixa etária, principais temas relatados, estratégias de enfrentamento e depoimentos espontâneos abordados no momento vivenciado.

A coleta das informações ocorreu em julho de 2010, tendo sido consultadas 775 fichas (100\%), dentre as quais foram selecionadas 110 (14\%) que apresentaram o registro do tema conflitos familiares. Para sistematizar o material empírico foi utilizado um instrumento elaborado para avaliar os impactos da $\mathrm{TCl}$ nas comunidades $^{(5)}$, o qual divide o tema em categorias analíticas e foi adaptado a este estudo.

Após a compilação sob aspectos de categorias analíticas, os dados foram submetidos ao tratamento da estatística simples e analisados a luz da literatura pertinente.

A pesquisa foi aprovada pelo Comitê de Ética em Pesquisa, do Centro de Ciências da Saúde da Universidade Federal da Paraíba - CEP/CCS, registrado sob protocolo $n^{\circ} 0197$ e consideram-se as observâncias éticas preconizadas na Resolução 196/96 do Conselho Nacional de Saúde (CNS), que dispõe sobre pesquisa em seres humanos no país.

\section{RESULTADOS}

Nas 775 fichas analisadas, foram encontradas 110 (14\%) situações que caracterizam diversos conflitos familiares. Dentre estes, a categoria de maior representatividade está relacionada aos cônjuges (48\%), seguidos dos conflitos geracionais entre pais e filhos (37\%), entre irmãos (8\%) e, por fim, entre avós e netos (7\%).

Foram selecionadas para análise as duas categorias de maior relevância que somam $85 \%$ das situações mencionadas pelos participantes, correlacionando-as com as temáticas que motivaram estes conflitos, tais como: desvalorização do papel da mulher, sobrecarga de trabalho, alcoolismo, separação, ciúmes, traição, dificuldade financeira, problemas intergeracionais, violência e drogadição.

Por meio dos registros de relatos dos participantes das rodas de $\mathrm{TCl}$, foi possível descrever as principais estratégias de enfrentamento das situações conflituosas, dentre as quais apontam: capacidade do autocontrole e autoconhecimento, suporte dos amigos e comunidade, participação em grupos de apoio, diálogo e espiritualidade.

\section{DISCUSSÃO}

A Estratégia Saúde da Família - ESF, concebida pela Política Nacional de Saúde, para fortalecimento da Atenção Básica oferecida pelo Sistema Único de Saúde SUS, à população brasileira, enfrenta, desde sua 
concepção, o desafio de acolher a grande demanda de pessoas que busca cuidados para promover a saúde e prevenir o adoecimento. Fortalecida pelo campo teórico da saúde coletiva, a ESF composta por equipes de profissionais, tais como: enfermeiros, dentistas e médicos, além dos agentes comunitários de saúde e outros profissionais de nível médio, oferecem serviços à comunidade visando atender as necessidades de saúde em nível primário.

Saindo da visão unilateral do processo saúde doença, as equipes da ESF buscam uma compreensão mais ampliada desse processo, onde os fatores biopsicossociais encontram-se intrinsecamente relacionados, compondo um jogo complexo de interações.

$\mathrm{Na}$ perspectiva de compreender as inter-relações que influenciam o adoecimento, é necessário romper com paradigmas reducionistas e fragmentadores, ou seja, sair da clínica individualizada para a ampliada e fazer uma leitura contextualizada na família e na sociedade, dentro de um sistema complexo de relações.

Nos últimos anos, a ESF vem enfrentando o desafio de acolher as pessoas em situação de sofrimento, seja por uma dor física ou emocional, sendo ofertados várias práticas e serviços à população, tais como: consulta individual, grupos de apoio e rodas de conversas, criando momentos de escuta, onde o profissional interage buscando encontrar as possíveis soluções para os problemas que emergiram.

Em um contexto onde a população vivencia cotidianamente situações de estresse, insegurança, perdas, abandono, carências materiais e afetivas, as equipes sofrem por se sentirem impotentes em face de problemáticas complexas e, geralmente, conduzem suas ações para estratégias pouco resolutivas, a exemplo da larga prescrição médica indicadora do uso de benzodiazepínicos, para problemas como falta de sono, dor de cabeça, dores nos ombros e inquietação.

Sem o poder de ampliar o tempo da escuta e potencializar os espaços de fala, vários profissionais da ESF encontraram na Terapia Comunitária Integrativa uma ferramenta capaz de otimizar e redimensionar a própria prática. A TCl criada pelo médico psiquiatra e antropólogo, Dr. Adalberto de Paula Barreto, na comunidade do Pirambú em Fortaleza-Ceará, Brasil, tem mais de 22 anos de prática, sendo aplicada em comunidades carentes, apresentando um impacto satisfatório quando se trata de potencializar espaços de fala e de escuta para que as pessoas possam encontrar soluções para os próprios problemas, sem necessariamente recorrer ao arsenal medicamentoso dos benzodiazepínicos ${ }^{(6,11,14)}$.

Em 2008 e 2009 a TCl foi implantada em 120 municípios brasileiros através de convênios com a UFC e Ministério da Saúde via Departamento de Atenção Básica em Saúde, para potencializar o trabalho da ESF. A avaliação do impacto desta ferramenta mostrou que a mesma reduziu em $89 \%$ os encaminhamentos para outros serviços ${ }^{(14-15)}$, evidenciando assim o efeito resolutivo da mesma, principalmente quando se trata de lidar com situações de sofrimento advindas de estressores presentes no cotidiano.

Nas primeiras análises do material documental desta pesquisa, chamou-nos atenção a variedade de temas vinculados ao sofrimento vivido no cotidiano das pessoas. Temas relacionados ao estresse, conflitos, drogas, depressão, trabalho e violência, foram compondo a nossa compreensão das tensões vividas no dia a dia dos indivíduos

Nesta perspectiva, as categorias encontradas na pesquisa representam situações comuns entre indivíduos que constituem uma família; e sendo esta um complexo sistema de relações interpessoais, foi necessário, em alguns momentos da análise, estabelecer associações entre as categorias.

A família é uma instituição que tanto influencia o todo e as partes, como sofre influencia destes, numa inter-relação contínua, configurando-se como um conjunto de indivíduos repletos de singularidades. Tendo em vista que a mesma é um sistema dinâmico, no qual os indivíduos estão constantemente resignificando seus papéis, o comportamento de seus componentes é influenciado pela maneira como são atribuídos as funções e os valores entres os membros ${ }^{(16)}$.

A categoria mais referida foi o conflito gerado entre os cônjuges, que pode ser caracterizada por situações provenientes de dificuldade financeira, constantes acusações, uniões marcadas por competição, agressões físicas ou dependência doentia de um ou de ambos os cônjuges. Porém, os motivos que mais se destacaram, segundo os participantes das rodas, foram a desvalorização da mulher perante o seu papel, a sobrecarga de atividades domésticas as quais thes foram atribuídas culturalmente, e o trabalho extra doméstico. 
Além desses, o alcoolismo do esposo e o ciúme possessivo para com a mulher, foram referidos como sendo, geralmente, intermediários para a violência conjugal. Frequentemente, esses conflitos são mais sentidos pelas mulheres por causar inquietações entre viver o amor próprio e manter um casamento sem amor. Outros fatores valorizados são o respeito pelos filhos, a dependência financeira, ou ainda, o medo de ser vítima de preconceito social.

A violência, sobretudo contra a mulher, expressa a desigualdade de poder nas relações de gênero, refletindo uma ordem patriarcal de organização familiar, constituindo-se como um problema de saúde pública. Embora a mulher esteja protegida legalmente, muitas preferem se submeter ao estado matrimonial, em silêncio, permeada pelo medo de sofrer outras agressões $^{(17)}$.

A Lei n 11.340, conhecida como Lei Maria da Penha, no seu Art. $5^{\circ}$, considera a violência doméstica aquela que acontece no espaço de convívio permanente de pessoas, com ou sem vínculo familiar, inclusive as esporadicamente agregadas; no âmbito da família, com indivíduos que são, ou se consideram, aparentados(18). Esta mesma lei coloca a violência conjugal como aquela que se dá em qualquer relação íntima de afeto, na qual o agressor conviva ou tenha convivido com a ofendida.

Esta categoria também está relacionada a separação matrimonial, a qual pode aflorar sentimentos de solidão pela perda da relação conjugal e convívio com os filhos. O divórcio apresenta-se como maior rompimento do ciclo da família e a tomada de uma nova direção, o que implica em reformulação de regras do funcionamento familiar e o desenvolvimento de novas capacidades adaptativas nos papéis que cada membro desenvolve ${ }^{(19)}$.

Geralmente, esse evento é sentido de forma negativa e traumático para os pais e filhos. Apesar do período de adaptação ser difícil, deve-se ressaltar que a separação pode ter aspectos positivos para todos os membros da família, sobretudo, quando se vivencia situações de violência física e verbal, ou quando um dos pais tem comportamentos antissociais, o que poderia trazer maiores intercorrências para o desenvolvimento do filho, caso os pais permanecessem unidos(19).

Outra causa geradora de conflitos entre cônjuges, frequentemente citada, foi a traição, evento referido, na maioria das vezes, como responsável pelas separações matrimoniais. A desagregação familiar é reflexo de uma sociedade liberal que favorece a fragilidade e a instabilidade da estrutura familiar, na qual percebe-se mudanças nos valores, existindo menos respeito e diálogo, o que suscita na criação de laços afetivos mais superficiais e na dissolução dos sentimentos, que regem as relações de família.

No tocante aos desentendimentos entre pais e filhos, percebeu-se que em sua maioria, eram originados pela dependência prolongada desses em relação aos pais. Essa situação, segundo relatos dos participantes, geralmente foi resultado do retorno de filhos casados ao domicílio familiar, devido ao desemprego, dificuldades financeiras ou separação conjugal.

Problema crônico e estrutural, o desemprego está presente nas sociedades contemporâneas, gerando tanto conflitos políticos, como problemas sociais e psicológicos ${ }^{(20)}$. São diversas as causas que levam ao desemprego, tais como a falta de qualificação dos trabalhadores face às exigências do mercado e a criação de tecnologias mais avançadas, que levam as empresas a substituir trabalhadores por equipamentos na produção.

Estar desempregado proporciona efeitos não só рага o individuo desempregado, mas também para todos os que o rodeiam, não se restringindo somente à perda de rendimentos ou poder de consumo, ou seja, não se limitando a dimensão pessoal(20).

Por outro lado, o retorno dos filhos à casa dos pais pode ser mola propulsora para relacionamentos conturbados entre sogras e noras/genros oriundos de uma relação de parentesco obrigatória e necessária, na qual estes são provenientes de famílias diferentes, possuindo hábitos, valores e crenças, muitas vezes, incompatíveis, dificultando o estabelecimento de vínculos. Pode ainda influenciar nessa relação a chegada de um filho/neto, pois a partir desse momento, poderão ter opiniões diferentes quanto à educação, uma vez que pertencem a diferentes gerações ${ }^{(21)}$.

Outra circunstância que ocasiona conflitos entre pais e filhos é a falta de incentivo aos jovens e adolescentes para os estudos e o desejo desses em adquirir bens de consumo mesmo sobre dificuldade financeira dos pais. Isto está relacionado, por um lado, à carência de orientação familiar, e por outro à fragilidade de instrumentos sociais, que ressaltem a valorização da escola como viés para uma vida social estável.

Estudos revelam que o enfraquecimento dos laços afetivos é um das consequências provenientes da 
competitividade impulsionada pelo capitalismo, uma vez que este cultua valores que vão de encontro às formas tradicionais de família. Dessa maneira, cria-se situações de isolamento, frustrações, que geram sentimentos de solidão, insegurança, baixa autoestima, favorecendo a comportamentos agressivos e também, a separação matrimonial(5). Essa conjuntura contribui para uma má qualidade de vida bem como, para marginalização e a exclusão de pessoas da sociedade.

As dificuldades financeiras e vulnerabilidade social são potenciais causas de conflitos familiares. Essa problemática é estimulada por uma sociedade excludente e de valores capitalistas que não absorve/acolhe os indivíduos e nem oferece oportunidade de fazê-los conquistar o que a sociedade indica como fator de inclusão. Esse jogo de valores sociais pode fazer o indivíduo envolver-se em condições vulneráveis como drogadição, e envolvimento com o crime.

Para lidar com os mais diversos conflitos, as famílias precisam utilizar de estratégias que proporcione a capacidade de desenvolver o autocontrole e manter relações permeadas pela afetividade e diálogo. No entanto, nem sempre seus membros possuem mecanismos eficazes para o enfrentamento de conflitos, o que potencializa a dimensão do sofrimento emocional. Por isso carece de atores envolvidos na luta pelo resgate e fortalecimento da instituição família, através da evidência de instrumentos como: suporte dos amigos e comunidade, implementação de atividades alternativas e de entretenimentos, a participação em grupos de apoio, o diálogo e a espiritualidade.

O autoconhecimento e autocontrole devem ser valorizados entre os membros da família, pois convida os indivíduos a refletir a respeito da necessidade de atitudes de cuidado e diálogo(22). Este reduziria significativamente esses conflitos, pois a necessidade da fala, do desabafo, da escuta, é de extrema importância para o entendimento da situação problema, encontrando através dele, meios para solução dos mesmos.

Nas relações interpessoais, percebe-se que a ajuda mútua contribuiu para que os envolvidos construam um olhar amplo no enfrentamento das dificuldades e de preocupação com o próximo. A partir disso se constroem as redes solidárias por conseguir consolidar os vínculos saudáveis, reforçar os vínculos frágeis e combater os vínculos de risco ${ }^{(13)}$.
A participação no grupo ajuda o indivíduo a se sentir mais integrado, uma vez que o ser humano é um ser gregário, estando inserido num grupo. Os grupos facilitam o exercício da autodeterminação e da independência, pois podem funcionar como rede de apoio que mobiliza as pessoas na busca de autonomia e sentido para a vida, na autoestima, na melhora do senso de humor, aspectos essenciais para se ampliar a resiliência e diminuir a vulnerabilidade ${ }^{(22)}$.

Com relação a participação em grupos de apoio, a partir de dispositivos de ajuda mútua, podem-se desenvolver atividades e iniciativas de cuidado e suporte concreto na vida cotidiana, como o cuidado informal ao outro, ajuda nas tarefas diárias. A ajuda mútua fornece apoio aos indivíduos na resolução de seus problemas, estabelece uma rede de amizade, que contribui, de maneira positiva, para a construção da autoimagem e eleva a autoestima ${ }^{(23)}$.

Nesse sentido, surge a Terapia Comunitária Integrativa que se constitui um espaço que permite o alívio do sofrimento, a construção de vínculos de amizade, de comunidade e vínculo social, onde as transformações provocadas revelam o fortalecimento e renovação de vínculos promotores de saúde, seja no espaço familiar, no lazer, e espiritual $(5,10)$.

Além disso, na $\mathrm{TCl}$ revela-se o empoderamento por meio da capacidade do participante em gerenciar a sua vida, e estimular a mobilização do grupo para questões inerentes ao coletivo, contribuindo para o surgimento da resiliência individual e coletiva ${ }^{(12)}$.

Dessa forma, a TCl contribui de maneira eficaz para o enfrentamento dos conflitos familiares cotidianos por possibilitar a escuta, a socialização dos problemas e estratégias. Os participantes descobrem que tem potencialidades para lidar com tais desafios, por meio do processo resiliente, de maneira eficaz, atuando nas relações interpessoais.

A partir das conversas na $\mathrm{TCl}$, a pessoa encontra dentro de si a resposta para suas inquietações e encontra apoio para superá-las. Então, partindo do princípio de que, no universo de mudanças rápidas e profundas, o importante não são os comportamentos corretos e fiéis ao esperado, mas sim o aumento da capacidade das pessoas/grupos para detectar os problemas reais e buscar-lhes solução original e criativa, a valorização de cada experiência se torna imperiosa. 
Constitui-se ainda, em relevante estratégia de inclusão da saúde mental na rede de cuidados primários à saúde, especialmente do cuidado a unidade familiar, sendo importante entender que a saúde mental nesse nível de atenção a saúde é complexa e requer atuação marcante quanto à responsabilidade sanitária no território e resolutividade frente às questões aqui apresentadas $^{(24)}$.

Nos encontros de Terapia Comunitária Integrativa, há momentos de intensa espiritualidade, com orações, imposição de mãos, respeitando-se as crenças e os valores de cada um. As práticas de cura pelo toque e pela imposição das mãos humanas como instrumento terapêutico tem sido usada, desde os primórdios da humanidade(25).

A espiritualidade ajuda o homem em sua compreensão, indo a sua esfera mais profunda, transcendendo a sua realidade. Entende-se espiritualidade como a arte e o saber que vai além das realidades habitualmente percebidas ${ }^{(23)}$. No enfrentamento dos problemas vivenciados no cotidiano, a fé, a crença em Deus é apontada como a estratégia mais utilizada, considerada, muitas vezes, a única ferramenta de resolução da dificuldade vivenciada.

Diante da fragilidade familiar encontrada no estudo, relacionado aos fatores responsáveis pela perda dos papeis familiares, pelo rompimento dos vínculos afetivos, pelo desrespeito entre os membros que culminam na ausência do diálogo, na dissolução de sentimento, que levam a traição dos cônjuges e até mesmo a separação matrimonial; a Terapia Comunitária Integrativa se tornou um relevante instrumento de desabafo e alívio dos sofrimentos, numa perspectiva resiliente para todos que venham a passar por situações semelhantes.

Percebe-se que a Terapia Comunitária Integrativa vem funcionando como base para o desenvolvimento de redes solidárias, reforço da identidade e diversidade cultural das comunidades, uma vez que trabalha perdas, crises, e valoriza as competências do indivíduo e da família ${ }^{(10,12)}$. Necessita, então, ser amplamente divulgada e valorizada como estratégia eficaz no enfrentamento de conflitos no meio familiar.

\section{CONCLUSÃO}

No presente estudo, verificou-se que os conflitos familiares estão significativamente presentes nos encontros de Terapia Comunitária Integrativa, gerando sofrimento emocional e risco de adoecimento mental, revelando fragilidade nos vínculos afetivos e de solidariedade. Dentre esses conflitos, o estudo apontou, sobretudo, discórdias que envolviam cônjuges e pais e filhos, devido a traição, alcoolismo, desvalorização do papel da mulher, sobrecarga de trabalho, separação, ciúmes, dificuldades financeiras, problemas intergeracionais, violência e drogadição, situaçõesproblemas que mais geram sofrimento e desagregação familiar.

Рага o enfrentamento dos conflitos familiares revelados, algumas estratégias foram sugeridas como o diálogo, apoio, a espiritualidade, a criação de um espaço de fala e escuta, o fortalecimento e/ou criação de vínculos afetivos e de solidariedade, mostrando que estes elementos estão um tanto ausentes no meio familiar, necessitando serem resgatados.

Faz-se necessária uma atenção especial para o papel familiar como base de formação dos seus membros e estrutura para relações sociais. Acrescenta-se que a estratégia da Terapia Comunitária Integrativa constituise em uma ferramenta para a consolidação dos vínculos familiares e de solidariedade, consistindo em um lócus, no qual essas estratégias de enfrentamento são fortalecidas.

Para o profissional da Atenção Básica de Saúde que lida cotidianamente com situações geradoras de sofrimento psíquico, é importante ter em mente que não existe bem-estar sem saúde mental, e que as pessoas devem ser valorizadas como instrumentos importantes para a construção de redes de apoio psicossocial na comunidade, tomando como base sua experiência de vida.

\section{REFERÊNCIAS}

1. Gomes NP, Diniz NMF, Araújo AJS, Coelho TMF. Compreendendo a violência doméstica a partir das categorias gênero e geração. Acta paul. enferm. [Internet]. 2007 [cited 2012 dec 31];20(4):504-8.

Available from: http://dx.doi.org/10.1590/S010321002007000400020 .
2. Clarke A. The sociology of healthcare. Londres: Pearson Education; 2001.

3. Oliveira D, Siqueira AC, Dell'Aglio DD, Lopes RCS. Impacto das Configurações Familiares no Desenvolvimento de Crianças e Adolescentes: Uma Revisão da Produção Científica. Interação em Psicologia [Internet]. 2008 [cited 2012 dec 31];12(1):87-98. Available 
from:

http://ojs.c3sl.ufpr.br/ojs2/index.php/psicologia/article/view/9172/ 9213.

4. Antoni C, Koller SH. A visão de família entre as adolescentes que sofreram violência intrafamiliar. Estud. psicol. (Natal) [Internet]. 2000 [cited $2012 \mathrm{dec} 31$ ]5(2):347-81. Available from: http://dx.doi.org/10.1590/S1413-294X2000000200004.

5. Barreto AP. Terapia comunitária passo a passo. Fortaleza: Gráfica LCR; 2008.

6. Ferreira Filha MO, Carvalho MAP. A terapia comunitária em um centro de atenção psicossocial: (des)atando pontos relevantes. Rev Gaucha Enferm [Internet]. 2010 [cited 2012 dec 31]; 31(2):232-9.

Available from: http://dx.doi.org/10.1590/S198314472010000200005 .

7. Rocha IA, Braga LAV, Tavares LM, Andrade FB, Ferreira Filha MO, Dias MD et al. A terapia comunitária como um novo instrumento de cuidado para saúde mental do idoso. Rev Bras Enferm [Internet]. 2009 [cited 2012 dec 31]; 62(5): 687-94. Available from: http://dx.doi.org/10.1590/S0034-71672009000500006. 8. Andrade FB, Ferreira Filha MO, Dias MD, Silva AO, Costa ICC, Lima EAR et al. Promoção da saúde mental do idoso na atenção básica: as contribuições da terapia comunitária. Texto Contexto Enferm [Internet]. 2010 [cited $2012 \mathrm{dec} 31$ ]; 19(1): 129-36. Available from: http://dx.doi.org/10.1590/S0104-07072010000100015.

9. Ferreira Filha MO, Dias MD, Andrade FB, Lima EAR, Ribeiro FF, Silva MSS. A terapia comunitária como estratégia de promoção à saúde mental: o caminho para o empoderamento Rev. Eletr. Enf. [Internet]. 2009 [cited $2012 \mathrm{dec} 31$ ];11(4):964-70. Available from: http://www.fen.ufg.br/revista/v11/n4/v11n4a22.htm.

10. Silva VCL, Souza GML, Silva PMC, Azevedo EB, Ferreira Filha MO, Espinola LL. A contribuição da terapia comunitária no processo saúde - doença. Cogitare Enferm [Internet]. 2011 [cited 2012 dec 31]; 16(4):682-8. Available from: http://ojs.c3sl.ufpr.br/ojs2/index.php/cogitare/article/view/23030.

11. Guimarães FJ, Ferreira Filha MO. Repercussões da terapia comunitária no cotidiano de seus participantes. Rev. Eletr. Enf. [Internet]. 2006 [cited $2012 \mathrm{dec} 31$ ];8(3):404-14. Available from: http://www.fen.ufg.br/revista/revista8_3/v8n3a11.htm.

12. Braga L, Dias MD, Ferreira Filha MO, Moraes MN, Araruna MHM, Rocha IA. Terapia Comunitária e resiliência: história de mulheres. R. pesq.: cuid. fundam. online [Internet]. 2012 [cited 2012 dec 31] Suppl:S251-68. Available from:

http://www.seer.unirio.br/index.php/cuidadofundamental/article/vi ew/1947/pdf 558.

13. Ferreira Filha MO, Sá ANP, Rocha IA, Silva VCL, Souto CMRM, Dias MD. Alcoolismo no contexto familiar: estratégias de enfrentamento das idosas usuárias da terapia comunitária. Rev. Rene [Internet]. 2012 [cited 2012 dec 31]; 13(1):26-35. Available from:

http://www.revistarene.ufc.br/revista/index.php/revista/article/vie $\underline{w / 13 / 10}$.

14. Araruna MHM, Ferreira Filha MO, Dias MD, Braga LAV, Moraes MN, Rocha IA. Formação de terapeutas comunitários na Paraíba: impacto na Estratégia Saúde da Família. Rev. Eletr. Enf. [Internet] 2012 [cited 2012 dec 31];14(1):33-41. Available from: http://www.fen.ufg.br/fen_revista/v14/n1/pdf/v14n1a04.pdf. 15. Brasil. Relatório Final da IV Conferência Nacional de Saúde Mental. Brasília: Ministério da Saúde; 2010.

16. Nunes ECDA, Silva LWS. A subjetividade de enfermeiros expressando em arte o significado do cuidado à família. Texto Contexto Enferm [Internet]. 2011 [cited 2012 dec 31];20(3): 453-60. Available from: http://dx.doi.org/10.1590/S010407072011000300005 .

17. Rabello PM, Caldas Júnior AF. Violência contra a mulher, coesão familiar e drogas. Rev Saude Publica [Internet]. 2007 [cited $2012 \mathrm{dec}$ 31];41(6):970-8. Available from: http://dx.doi.org/10.1590/S003489102007000600012 .

18. Lei n. 11.340, de 7 de agosto de 2006 (BR). Cria mecanismos para coibir a violência doméstica e familiar contra a mulher, nos termos do § 80 do art. 226 da Constituição Federal, da Convenção sobre a Eliminação de Todas as Formas de Discriminação contra as Mulheres e da Convenção Interamericana para Prevenir, Punir e Erradicar a Violência contra a Mulher; dispõe sobre a criação dos Juizados de
Violência Doméstica e Familiar contra a Mulher; altera o Código de Processo Penal, o Código Penal e a Lei de Execução Penal; e dá outras providências. Diário Oficial da União [Internet]. 05 aug 1994 [cited 2012 dec 31]. Available from:

http://www.planalto.gov.br/ccivil_03/_ato20042006/2006/lei/l11340.htm.

19. Cano DS, Gabarra LM, Moré CO, Crepaldi MA. As transições familiares do divórcio ao recasamento no contexto brasileiro. Psicol. Refl. Crít. [Internet]. 2009 [cited 2012 dec 31];22(2):214-22.

Available from: http://dx.doi.org/10.1590/S0102$\underline{79722009000200007 .}$.

20. Gondim SMG, Estramiana JLA, Luna AF, Oliveira TSS, Souza GC. Atribuições de causas ao desemprego e valores pessoais. Estud. psicol. (Natal) [Internet]. 2010 [cited 2012 dec 31];15(3):309-17. Available from: http://dx.doi.org/10.1590/S1413294X2010000300011.

21. Dias CMS, Hora FFA, Aguiar AGS. Jovens criados por avós e por um ou ambos os pais. Psic.: Teor. e Pesq. [Internet]. 2010 [cited 2012 dec 31];12(2):188-99. Available from: http://pepsic.bvsalud.org/pdf/ptp/v12n2/v12n2a13.pdf.

22. Martins JJ, Barra DCC, Santos TM, Hinkel V, Nascimento ERP, Albuquerque GL, et al. Educação em saúde como suporte para a qualidade de vida de grupos da terceira idade. Rev. Eletr. Enf. [Internet]. 2007 [cited $2012 \mathrm{dec} 31$ ];9(2):443-56. Available from: http://www.fen.ufg.br/revista/v9/n2/v9n2a12.htm.

23. Vasconcelos EM. A espiritualidade no trabalho em saúde. São Paulo: Hucitec; 2006

24. Botti, NCL, Andrade WV. A saúde mental na atenção básicaarticulação entre os princípios do SUS e da reforma psiquiátrica. Cogitare Enferm [Internet]. 2008[cited 2012 dec 31]; 3(3):387-94. Available from: http://ojs.c3sl.ufpr.br/ojs2/index.php/cogitare/article/view/12991. 25. Marta IER, Baldan SS, Berton AF, Pavam M, Silva MJP. Efetividade do Toque Terapêutico sobre a dor, depressão e sono em pacientes com dor crônica: ensaio clínico. Rev Esc Enferm USP [Internet]. 2010 [cited 2012 dec 31];44(4):1100-6. Available from: http://dx.doi.org/10.1590/S0080-62342010000400035.

Artigo recebido em 20/09/2011. Aprovado para publicação em 02/07/2012. Artigo publicado em 31/12/2012. 